\title{
In Vivo Translation of the CIRPI System: Revealing Molecular Pathology of Rabbit Aortic Atherosclerotic Plaques
}

\author{
Raiyan T. Zaman ${ }^{1-3}$, Siavash Yousefi ${ }^{4}$, Hidetoshi Chibana ${ }^{3}$, Fumiaki Ikeno ${ }^{3}$, Steven R. Long ${ }^{5}$, Sanjiv S. Gambhir ${ }^{6-8}$, \\ Frederick T. Chin ${ }^{6,7}$, Michael V. McConnell ${ }^{3,7,9}$, Lei Xing ${ }^{4,7}$, and Alan Yeung ${ }^{3}$ \\ ${ }^{I}$ Department of Radiology, Harvard Medical School, Boston, Massachusetts; ${ }^{2}$ Gordon Center for Medical Imaging, Massachusetts \\ General Hospital, Boston, Massachusetts; ${ }^{3}$ Division of Cardiovascular Medicine, Department of Medicine, Stanford University \\ School of Medicine, Stanford, California; ${ }^{4}$ Division of Medical Physics, Department of Radiation Oncology, Stanford University \\ School of Medicine, Stanford, California; ${ }^{5}$ Department of Pathology, Stanford University School of Medicine, Stanford, California; \\ ${ }^{6}$ Department of Radiology, Stanford University School of Medicine, Stanford, California; ${ }^{7}$ Molecular Imaging Program at Stanford \\ University (MIPS), Stanford University School of Medicine, Stanford, California; ${ }^{8}$ Department of Bioengineering, Stanford University \\ Schools of Medicine and of Engineering, Stanford, California; and ${ }^{9}$ Verily Life Sciences, San Francisco, California
}

Thin-cap fibroatheroma (TCFA) are the unstable lesions in coronary artery disease that are prone to rupture, resulting in substantial morbidity and mortality worldwide. However, their small size and complex morphologic and biologic features make early detection and risk assessment difficult. We tested our newly developed catheter-based Circumferential-Intravascular-RadioluminescencePhotoacoustic-Imaging (CIRPI) system in vivo to enable detection and characterization of vulnerable plaque structure and biology in rabbit abdominal aorta. Methods: The CIRPI system includes a novel optical probe combining circumferential radioluminescence imaging and photoacoustic tomography (PAT). The probe's $\mathrm{CaF}_{2}$ : Eu-based scintillating imaging window captures radioluminescence images $\left(360^{\circ}\right.$ view) of plaques by detecting $\beta$-particles during ${ }^{18} \mathrm{~F}$-FDG decay. A tunable laser-based PAT characterizes tissue constituents of plaque at 7 different wavelengths -540 and 560 $\mathrm{nm}$ (calcification), $920 \mathrm{~nm}$ (cholesteryl ester), $1040 \mathrm{~nm}$ (phospholipids), $1180 \mathrm{~nm}$ (elastin/collagen), $1210 \mathrm{~nm}$ (cholesterol), and $1235 \mathrm{~nm}$ (triglyceride). A single B-scan is concatenated from 330 A-lines captured during a $360^{\circ}$ rotation. The abdominal aorta was imaged in vivo in both atherosclerotic rabbits (Watanabe Heritable Hyper Lipidemic [WHHL], 13-mo-old male, $n=5$ ) and controls (New Zealand White, $n=2$ ). Rabbits were fasted for $6 \mathrm{~h}$ before $5.55 \times 10^{7}$ $\mathrm{Bq}(1.5 \mathrm{mCi})$ of ${ }^{18} \mathrm{~F}-\mathrm{FDG}$ were injected $1 \mathrm{~h}$ before the imaging procedure. Rabbits were anesthetized, and the right or left common carotid artery was surgically exposed. An 8 French catheter sheath was inserted into the common carotid artery, and a $0.035-\mathrm{cm}$ (0.014-in) guidewire was advanced to the iliac artery, guided by $\mathrm{x}$-ray fluoroscopy. A bare metal stent was implanted in the dorsal abdominal aorta as a landmark, followed by the 7 French imaging catheters that were advanced up to the proximal stent edge. Our CIRPI and clinical optical coherence tomography (OCT) were performed using pullback and nonocclusive flushing techniques. After imaging with the CIRPI system, the descending aorta was flushed with contrast agent, and OCT images were obtained with a pullback speed of $20 \mathrm{~mm} / \mathrm{s}$, providing images at 100 frames/s. Results were verified with histochemical analysis. Results: Our CIRPI system successfully detected the locations and characterized both stable

Received Oct. 23, 2018; revision accepted Jan. 13, 2018.

For correspondence or reprints contact: Raiyan T. Zaman, Department of Radiology, Harvard Medical School, Gordon Center for Medical Imaging, Massachusetts General Hospital, 149 13th St., Charlestown, MA 02129.

E-mail: rzaman@mgh.harvard.edu

Published online Feb. 8, 2019.

COPYRIGHT (c) 2019 by the Society of Nuclear Medicine and Molecular Imaging. and vulnerable aortic plaques in vivo among all WHHL rabbits. Calcification was detected from the stable plaque $(540$ and $560 \mathrm{~nm})$, whereas TCFA exhibited phospholipids/cholesterol (1040 nm, 1210 $\mathrm{nm})$. These findings were further verified with the clinical OCT system showing an area of low attenuation filled with lipids within TCFA. PAT images illustrated broken elastic fiber/collagen that could be verified with the histochemical analysis. All WHHL rabbits exhibited sparse to severe macrophages. Only 4 rabbits showed both moderate-to-severe level of calcifications and cholesterol clefts. However, all rabbits exhibited broken elastic fibers and collagen deposition. Control rabbits showed normal wall thickness with no presence of plaque tissue compositions. These findings were verified with OCT and histochemical analysis. Conclusion: Our novel multimodality hybrid system has been successfully translated to in vivo evaluation of atherosclerotic plaque structure and biology in a preclinical rabbit model. This system proposed a paradigm shift that unites molecular and pathologic imaging technologies. Therefore, the system may enhance the clinical evaluation of TCFA, as well as expand our understanding of coronary artery disease.

Key Words: cardiology (basic/technical); instrumentation; molecular imaging; optical; optical imaging; photoacoustic imaging; radioluminescence imaging; TCFA; multi-mode catheter based imaging system

J Nucl Med 2019; 60:1308-1316

DOI: 10.2967/jnumed.118.222471

A cute coronary syndrome leads to a sudden reduction in perfusion and oxygenation to the myocardium resulting in heart attack. Each year, more than 20 million people with coronary artery disease (CAD) experience acute coronary syndrome, and one third of them die from complications (1). The underlying causes of acute coronary syndrome are unstable lesions known as thin-cap fibroatheroma (TCFA), which are prone to rupture, resulting in substantial morbidity and mortality worldwide. This is a progressive condition typically asymptotic at early stages and is characterized by the thickening of the arterial vessel wall due to the buildup of arthermanous plaque in the inner lining of the arteries $(2,3)$.

Early clinical diagnosis of TCFA is thought to be critical not only to prevent catastrophic events of plaque rupture through intervention or pharmacologic strategy but also to contribute to the 
study of epidemiology of vulnerable plaques. Although the current clinical definition of TCFA is large lipid pool, heavy infiltration of inflammatory cells/macrophages, and fibrous wall thickness less than $65 \mu \mathrm{m}$, understanding TCFA, the most common phenotype of vulnerable plaques, remains to be elucidated. For the larger-sized coronary arteries, various intravascular imaging modalities have been developed and tested clinically, including optical coherence tomography (OCT) and intravascular ultrasound (4-7). Although these methods provide information about the structural characteristics of the imaged vessel, they do not directly characterize biologic aspects of disease $(8,9)$, such as inflammation or angiogenesis $(10,11)$. There is a wide range of molecular imaging modalities (such as iodinated nanoparticulate contrast agent, photoacoustic imaging using spheric nanoparticles (12) and gold nanorods (13), intravascular photoacoustic (IVPA) with optical contrast (14), MRI with wall sheer stress (15), CT with N1177 (16), PET with ${ }^{18}$ F-FDG (5), MRI using ultrasmall superparamagnetic iron oxide probes $(17,18)$, SPECT using VCAM-1-specific ${ }^{99 m}$ Tc-labeled peptidic sequences (19), hybrid imaging such as simultaneous OCT for $\beta$-detection (20), OCT-near-infrared fluorescence system with indocyaninegreen contrast agent used for colocalization of anatomic and molecular information from the artery wall). However, because of their small size, motion, and obscuring signal from the adjacent myocardium, it is still difficult to detect TCFA in the early stages. Therefore, an optimal intravascular imaging technology for the identification of TCFA needs to possess unique features such as visualizing endoluminal structure (2), identifying plaque components (21), providing adequate spatial resolution, and monitoring plaque rupture/thrombus formation. However, because of the inherent limitations of these currently available imaging systems, an integration of multiple imaging modalities seems a synergistic solution $(22,23)$.

To address these limitations, we developed a multimode Circumferential-Intravascular-Radioluminescence-Photoacoustic-Imaging (CIRPI) system that would permit the precise location and evaluation of coronary vulnerable plaque pathology in an early stage (24). The goal of this feasibility study was to test the CIRPI system in in vivo an atherosclerotic rabbit model to detect the TCFA with superior spatial resolution (25) and improve the quantification of aortic TCFA morphology relative to other imaging modalities that are clinically available today. The innovation of this study was both technologic and intellectual novelty as it aimed to distinguish TCFA from stable plaques with high sensitivity and accuracy and provide quantitative image maps of TCFA with colocalized information on plaque compositions with histopathologic information $(24,26)$. In this study, we present the in vivo rabbit experiments performed with our CIRPI system to monitor disease compositions and location through mapping perturbed microanatomic features of TCFA.

\section{MATERIALS AND METHODS}

\section{Circumferential Radioluminescence Imaging (CRI) Peripheral System Design}

The components of the CIRPI system are described in our previously published study (26). In brief, the optical components of the CIRPI system consist of a $10 \times$ magnification infinity-corrected microscope objective (RMS10x; Olympus Inc.) with a working distance of $10.6 \mathrm{~mm}$, numerical aperture (NA) of 0.25 , and effective focal length of $18 \mathrm{~mm}$ (Supplemental Fig. 1; supplemental materials are available at http:// jnm.snmjournals.org). The system also contains an infinity-corrected tube lens for plan fluorite objective (ITL200; Thorlabs) in between the objective $(\mathrm{F} 2=102 \mathrm{~mm})$ and the ProEM charge-coupled device
(CCD) camera $(\mathrm{F} 3=200 \mathrm{~mm}$ ) (Princeton Instruments). The CIRPI system provides a $360^{\circ}$ view inside an artery through an innovative probe design (see the "Dual-Modality Endoscopic Probe" section).

The novelty of this CIRPI system is based on a scintillating window made from organic calcium fluoride doped with europium $(\mathrm{CaF} 2 \mathrm{Eu})$ phosphor, placed on the catheter-based probe to convert the $\beta$-particles of the positron emission signal into visible light due to radioactive decay (primary decay time $940 \mathrm{~ns}$ ). The molecules in the scintillating window get excited with the incoming ionizing radiation, especially $\beta$-particles from the decay of ${ }^{18} \mathrm{~F}-\mathrm{FDG}$, or other isotopes, by absorbing energy. When these molecules return to a lower energy state from an excited state, they release optical radiation in the visible range. This light is then captured with a highly sensitive CCD camera using deep thermoelectric cooling at $-70^{\circ} \mathrm{C}$ for minimizing the background signal from the temperature-dependent dark current, hot pixel blemishes, and vibration. The deep thermoelectric cooling was done with a CoolCUBE II liquid circulatory system (Teledyne Princeton Instruments). The camera exposure times were set to $45 \mathrm{~s}$ based on sensitivity (above this threshold the pixels were saturated). For ${ }^{18}$ F-FDG imaging with the CIRPI system, the binning factor was set at the smallest limit $(1 \times 1$ pixel) with effective active imaging resolutions of $1,024 \times 1,024$ pixels (width $\times$ height). The analog-to-digital conversion speed was set to $10 \mathrm{MHz}$, with a corresponding electron multiplying gain of 50 at 16-bit depth. The storage shift rate was set to $600 \mathrm{~ns}$ with readout time of $4 \mathrm{~ms}$ for "frame transfer" readout mode. The CCD camera shutter mode was set to "always open" without any delay $(0 \mathrm{~ms})$ in opening and closing. The selection process of optimal scintillating material and resolution were described in the our previously published manuscript (25).

\section{Photoacoustic Tomography (PAT) Peripheral System Design}

The PAT peripheral system has been redesigned to perform faster data acquisition from our previously published study (24). For the PAT system, we used a tunable Horizon I OPO pumped by Surelite II nanosecond laser (192-2,750 nm Continiuum) with a 4- to 7-mm beam diameter (before focusing into the $200-\mu \mathrm{m}$ core multimode fiber). The peak wavelength of the laser's spectral bandwidth can be tuned to multiple values for selective imaging of the different tissue constituents. The central hypothesis of this PAT imaging is that the absorption spectra of calcium, lipids, elastic, and collagen are sufficiently well differentiated from those of the constituents of normal arterial tissue in the 500- to 1400 -nm wavelength range to permit the detection of calcium or lipid-rich plaques using PAT imaging.

The repetition rate was set to the highest at $20 \mathrm{~Hz}$ with a 3- to 5-ns full width at half maximum pulse length. The laser was linearly polarized at a less than 2 mrad divergence. The Horizon tunable laser operates at $110 \mathrm{~V}$ and is connected to Surelite 210. Power supply for Surelite 210 operates at $220 \mathrm{~V}$ and is attached to a coolant circulation to regulate the temperature. Horizon sends an Sync Out signal as an external trigger to a pulse-receiver signal generator (5073PR-15U, 75 MHz bandwidth, 39dB, RF Gain, 115 VAC; Olympus America Inc.). A preamplifier receives the photoacoustic signal from the multimode optical probe, and after $8 \times$ amplification this signal is sent to the pulsereceiver. This $75 \mathrm{MHz}$ computer-controlled pulse-receiver is used to trigger a 4-channel, 14-bit Digital Acquisition System (NI PXIe5170R, $250 \mathrm{MS} / \mathrm{s}, 100 \mathrm{MHz}$, Oscilloscope with $750 \mathrm{MB}$ RAM and Kintex-7 325T FPGA; National Instruments, USA) and receive the amplified photoacoustic signal. A custom-written Matlab (The MathWorks) code was used to concatenate 330 A-lines captured during a $360^{\circ}$ rotation to generate a B-scan.

\section{Dual-Modality Endoscopic Probe}

The components of CIRPI system probe are described in our previously published study (26). However, the probe is reduced in diameter. The CIRPI system includes a novel optical probe combining 
CRI and PAT (Supplemental Fig. 2A). The probe's CaF2:Eu-based scintillating imaging window captures radioluminescence images $\left(360^{\circ}\right.$ view) of plaques by converting $\beta$-particles to visible photons during ${ }^{18}$ F-FDG decay (Supplemental Fig. 2B). A single $360^{\circ}$ arterial radioluminescence image acquisition is $45 \mathrm{~s}$ for our CIRPI system. During radioluminescence imaging, the visible photons from the optical radiation of high-energy $\beta$-particles reflect to/from the $45^{\circ}$ scanning mirror. These photons are then collected through the gradient-index (GRIN) lens followed by creating an 18,000-pixel image at every $1.43^{\circ}$ rotation of the mirror. A total of 63 images were collected for each quadrant of the circular arterial wall. A tunable laser-based PAT characterizes tissue constituents of plaque at 7 different wavelengths - 540 and $560 \mathrm{~nm}$ (calcification), $920 \mathrm{~nm}$ (cholesteryl ester), $1040 \mathrm{~nm}$ (phospholipids), $1180 \mathrm{~nm}$ (elastin/collagen), $1210 \mathrm{~nm}$ (cholesterol), and $1235 \mathrm{~nm}$ (triglyceride) (Supplemental Fig. 2C).

The CIRPI probe (Supplemental Figs. 2D and 2E) has a $840-\mathrm{mm}$ flexible tether with up to $17^{\circ}$ of bending freedom, and a 36-mm (previously $42 \mathrm{~mm}$ ) long rigid distal imaging head consists of a CaF2:Eu scintillating imaging window; a light-guiding multimode optical fiber (OF-1, 0.22 NA, $0.2 \mathrm{~mm}$ core diameter; Thorlabs Inc., USA); a leached image fiber (OF-2, 18,000 optical fibers represent a 0.9-mm diameter imaging area with 7.4- $\mu \mathrm{m}$ pixel size; Schott Inc., USA); a single-element ultrasonic transducer (Lithium Niobate LNO, 40 $\mathrm{MHz}$, unfocused, ring transducer with outer diameter $=2 \mathrm{~mm}$, inner diameter $=1.37 \mathrm{~mm}$, and length $=5.1 \mathrm{~mm}$ ); a digital actuator (2.0-mm diameter, $18.62 \mathrm{~mm}$ long; Namiki Precision Jewel Co., Ltd. Japan); and $45^{\circ}$ degree flat rotating mirror (2-mm diameter, protected aluminum on glass substrate, with the reflection surface at $45^{\circ}$ to the probe's axis; Edmund Optics Inc., USA) that is placed in a stainless steel tube housing. The absolute field-of-view of $0.9-\mathrm{mm}$ imaging area is $6.5 \mathrm{~mm}$. A single-element ultrasonic transducer is used for achieving a high signal to noise ratio at $\mathrm{MHz}$ repetition rates. Laser pulses from a portable UV-VIS-NIR tunable laser (7 ns pulse length at $20 \mathrm{~Hz}$ repetition rate) are coupled into a multimode fiber using a $10 \times$ microscope objective. These laser pulses are guided by the multimode optical fiber, which is also positioned parallel to an imaging fiber-bundle belonging to the CIRPI system. These pulses are then emitted into the tissue through a central hole $(1.37-\mathrm{mm}$ diameter) in the transducer located in the distal end of the probe. The distal end of the OF-2 is terminated by a $1-\mathrm{mm}$ diameter GRIN lens designed to have a working distance of $5 \mathrm{~mm}$ and paraxial magnification of 5.86 . The GRIN lens provides an NA of 0.5 , and radial index gradient has a maximum central refractive index of 1.635 at the lens axis.

The scintillating window is fabricated from a polymer-based polyvinyl toluene block made of $\mathrm{CaF}$ :Eu phosphor (Saint-Gobain Crystals). $\mathrm{CaF} 2 \mathrm{Eu}$ is selected as the optimal scintillating material with respect to highest radioluminescent signal (25). The phosphor has a refractive index of 1.58 , with $68 \%$ light output. The scintillating efficiency is 10,400 photons $/ 1 \mathrm{MeV}$ - - It has a maximum emission at a 435 -nm wavelength with a temporal pulse width of $2.2 \mathrm{~ns}$ (full width at half maximum). The scintillating window is machined into a cylindrically shaped tube with a $10-\mathrm{mm}$ length and wall thickness of $0.150 \mathrm{~mm}$. The outer diameter and inner diameter of the window are 2.31 and $2.01 \mathrm{~mm}$, respectively. Radioluminescence is produced within the scintillating imaging window following the emission of a $\beta$-particle from a radiotracer $\left({ }^{18} \mathrm{~F}-\mathrm{FDG}\right)$ within a macrophage. The optical photons are captured by a high-numericalaperture $10 \times$ microscope objective coupled to a deep-cooled ProEM CCD camera. This scintillating window can detect radioluminescent signal from the disease tissue within the travel distance of $\beta$-particles (1-3 mm in tissue) emitted from the ${ }^{18} \mathrm{~F}-\mathrm{FDG}$ decay. The CRI peripheral system provides the overall spatial resolution of the CIRPI system that is $1.2 \mu \mathrm{m}$ based on our previous study (25). The spatial resolution of the CRI is calculated on the basis of a modulation transfer function. A modulation transfer function is an important aid to objective evaluation of the image-forming capability of our CRI peripheral system, providing a means of expressing the imaging quality of the optical system objectively and quantitatively, plus predicting performance reliably. The detection limit and overall radioluminescence signal sensitivity of our system is identified to be 10,020 (photon counts) on the basis of a $1-\mu \mathrm{Ci}$ closed disk $\beta$-source.

Circumferential sector scanning (B-scan) is accomplished by rotating a $45^{\circ}$ flat mirror. The mirror is driven by a geared actuator (gear ratio, 254:1) to steer the laser beam from the optical fiber to the tissue and the acoustic wave from the tissue to the ultrasonic transducer. A single $360^{\circ}$ arterial PAT image acquisition time is $1.15 \mathrm{~s}$ for our CIRPI system. Therefore, the overall detection speed for a $360^{\circ}$ view of an artery with our CIRPI system took only a few minutes $(\approx 2 \mathrm{~min})$. When the pulsed laser light illuminates atherosclerotic plaque, the optical absorbers there (such as lipid, cholesterol, calcification) undergo thermoelastic expansion, generating an acoustic pressure wave that is detected with the ultrasonic transducer. The mirror is also important for reflecting visible light to the OF-2 due to the vibration of molecules within the scintillating imaging window upon the deposition of $\beta$-particles energy from the ${ }^{18}$ F-FDG decay. As water and glass have a large ratio of sound propagation speeds, the scanning mirror has exhibited total external reflection within the acceptance angles of the ultrasonic transducer and the GRIN lens, and thus, contributes to no additional propagation losses into the ultrasonic and visible light detection. The scanning mirror system has enabled circumferential B-scanning without moving other illumination optics and the ultrasonic sensor. The optical fibers, the transducer's signal wires, and the actuator wires are encapsulated in a flexible catheter body with an outer diameter of $2.31 \mathrm{~mm}$ where $0.5 \mathrm{~mm}$ is used for a catheter enclosure. The mirror's rotational speed is kept constant, while providing a matching medium for acoustic wave propagation; the transducer and scanning mirror's housing space is water-coupled with deionized water through a 0.25 -mm hole on the 0.150 -mm-thick scintillating imaging window. The actuator is isolated from the water with a spacer, and the torque is required for the mirror rotation that is transferred through a micro-magnetic (outer diameter, $1.58 \mathrm{~mm}$ with a length of $3.175 \mathrm{~mm}, \mathrm{~K} \& \mathrm{~J}$ Magnets Inc., USA) coupling mechanism.

\section{PAT Image Reconstruction}

PAT images are created from the generation of thermoelastic stress waves by heating endogenous chromophores (e.g., calcification, cholesterol) or exogenous contrast agents in an optically heterogeneous medium with a short laser pulse. These stress waves contain information about the distribution of structures with preferential optical absorption. Therefore, information about the relative concentrations of endogenous chromophores or exogenous contrast agents can be determined allowing enhanced signal-to-background ratios. The low optical absorption and ultrasound scattering of disease tissues make it optimal for PAT imaging at high resolution and depth (maximum, $3-5 \mathrm{~cm}$ deep). However, for this study we adjusted the depth to be between 5 and $8 \mathrm{~mm}$.

The temporarily confined optical absorption induces a temperature rise $\Delta \mathrm{T}$ and consequently an initial pressure rise $\mathrm{p} 0$ due to thermoelastic expansion: $\mathrm{p} 0=\beta . \Delta \mathrm{T} / \mathrm{k}$, where $\mathrm{k}$ is the isothermal compressibility. The photoacoustic signal was collected every $1.43^{\circ}$ rotating step of the scanning mirror for photoacoustic acquisition at 7 different wavelengths. The PAT image is developed one laser pulse per image line. The procedure of photoacoustic signal processing includes highpass signal filtering, compensation for acoustic diffraction, detection of the irradiated surface position, and rejection of the reverberating signal. A radial backprojection algorithm for image reconstruction is developed and included in the Labview/Matlab computer code. Laser-induced noise is removed with a singular value decomposition method. 


\section{Coregistered with Histologic Analyses and OCT}

After the in vivo imaging experiments, WHHL $(n=5)$ and New Zealand White (NZW) $(n=2)$ rabbit dorsal abdominal aortic samples were cut from the distal edge of the stent (used as a landmark) and placed in $10 \%$ formalin and submitted for paraffin embedding, sectioning, and staining. Step sections ( $5 \mu \mathrm{m}$ thick, $n=16)$ were collected at different levels beginning at the proximal end of the stent to exclude the aorta-containing stent (otherwise the blade would brake) and proceeding to the other end. The sections were mounted on glass microscopic slides, stained with hematoxylin and eosin (H\&E), trichrome (collagen), and EVG (elastin) reagents and covered with Tissue Tek Film as coverslips.

The microscopic slides were examined with an Olympus BX51 microscope fitted with diffuse white light (LM) and transmission polarizing (TPM) optics and $2 \times, 4 \times, 10 \times, 20 \times$, and $40 \times$ objectives. Selected fields were imaged by a pathologist at Stanford SOM using a Zeiss Axiocam MRc5 digital camera mounted on the microscope and Zeiss AxioVision SE64 Rel 4.8 software supported by a Dell Optiplex 980 desktop computer. The histologic study was based on a quantitative analysis of disease tissue constituents that were coregistered with the CIRPI results and clinical OCT based on the stent location.

\section{Safety Assurance}

After each experiment, we used a classic $\gamma$-counting analog meter (Geiger-Muller counter) to perform safety assurance on the instruments, countertop, whole body, and laboratory coat, to ensure that there was no contamination from ${ }^{18} \mathrm{~F}-\mathrm{FDG}$. Each time, the counts per minute were around 30-50 CPM, which was in the normal range of $0.03-0.05 \mathrm{mR} / \mathrm{hr}$.

\section{Statistical Analysis}

A pairwise 2-sample Student $t$ test was performed to compare in vivo radioluminescent signal intensity from the ${ }^{18} \mathrm{~F}$-FDG-enriched vulnerable plaques and stable plaques. The same $t$ test was performed for photoacoustic signals as well for both TCFA and non-TCFA. As WHHL rabbits had similar weights $(2.27 \pm 0.01 \mathrm{~kg}[5 \pm 0.02 \mathrm{lb}])$ and the same dose, these factors were not considered in the statistical analysis. Therefore, weight and dose were eliminated during the statistical process. Rabbit samples were not randomized. These analyses were performed using custom-written MATLAB code. We presented all values as mean $\pm \mathrm{SD}$. We considered a $P$ value of less than 0.05 as statistically significant for all ex vivo analyses.

For a significance level of $P<0.05$ and a statistical power of 0.90 , each group should comprise at least 5 animals to obtain a statistically significant result. We used an equation for sample size calculation for a study comparing 2 means: $N=\{4 * \sigma 2($ Zcrit + Zpwr)2 $\} / D 2$, where $\mathrm{N}$ is sample size, $\mathrm{D}$ is the minimum expected contrast-to-noise difference based on the means of the 2 groups (plaque vs. control), $\sigma$ is the assumed SD of each group (assumed to be equal for both group) $=1.65$, Zcrit $=1.960$ for a significance level of 0.05(95) (standard normal deviate corresponding to selected significance criteria), and Zpwr $=1.645$ for statistical power of 0.95 (standard normal deviate corresponding to selected statistical powers).

\section{Experimental Procedure of In Vivo Imaging of Atherosclerotic Plaque}

We tested this system in in vivo rabbit (13-mo-old male; $n=5$, WHHL; $n=2$, NZW, control) abdominal aorta models according to an approved protocol by the Stanford University Administrative Panel on Laboratory Animal Care (APLAC 9948). The WHHL rabbit model is hypercholesterolemic due to a deficiency of low-density lipoprotein receptors and has contributed to studies about lipoprotein metabolism, hypercholesterolemia, and atherosclerosis. We let the WHHL rabbits acclimate for $7 \mathrm{~d}$ after arrival from the Institute for Experimental Animals, Kobe University Graduate School of Medicine. The same acclimation procedure was followed for the NZW rabbits (Charles River Laboratories, USA). After 7 d, we performed preanesthetic evaluations of rabbits by chest auscultation to determine whether the rabbits were suitable candidates for the imaging procedure. Once determined, rabbits were fasted for $6 \mathrm{~h}$ before preoperative care was provided to them $30 \mathrm{~min}$ before surgery and $5.55 \times 10^{7} \mathrm{~Bq}$ of ${ }^{18} \mathrm{~F}$-FDG were injected $1 \mathrm{~h}$ before imaging.

During the procedure, rabbits were anesthetized with ketamine, xylazine, bupivacaine, and maintained with inhaled isoflurane with $3 \%$ oxygen through intubation. Hair in the neck area was shaved, and $1 \%$ lidocaine was applied to vocal folds with a cotton-tipped applicator to facilitate intubation with an endotracheal tube to maintain airways and prevent laryngospasm. A midline neck incision was made to surgically expose right or left the common carotid artery $(2.85 \pm$ $0.15 \mathrm{~mm}$ in diameter) (Supplemental Fig. 3A). An 8 French (2.67$\mathrm{mm}$ diameter) catheter sheath was inserted into the common carotid artery carefully, and a $0.035 \mathrm{~cm}(0.014 \mathrm{in})$ guidewire was advanced into the common carotid artery followed by an implantation of baremetal stent in the dorsal abdominal aorta $(4 \pm 0.38 \mathrm{~mm}$ diameter $)$ as a landmark. Then a 7 French $(2.31 \mathrm{~mm}$ in diameter $)$ imaging catheter was inserted into the descending aorta guided through $\mathrm{x}$-ray fluoroscopy (Supplemental Fig. 3B). The CIRPI probe needed to be within $1-3 \mathrm{~mm}$ of the plaques for the radioluminescence image. However, for this experiment the probe was within $0.75 \mu \mathrm{m}$ from the arterial wall (based on acoustic signal). Our CIRPI and clinical OCT (ILUMEN OCT Imaging System; Abbott) were performed using pullback and nonocclusive flushing techniques. After imaging with the CIRPI system, the descending aorta was flushed with contrast agent, and OCT images were obtained with a pullback speed of $20 \mathrm{~mm} / \mathrm{s}$, providing images at 100 frames/s. Results were verified with histochemical analysis.

\section{RESULTS}

In Figures 1-3, images from 1 control rabbit (ID\#R817) and 1 WHHL (ID\#R717) are shown for discussion purpose only. The CIRPI and confirmatory clinical OCT imaging were done at the proximal stent edge located within the abdominal aorta of the control rabbit (highlighted with a green box), performed under the x-rayguided fluoroscopy (Fig. 1A). The H\&E-stained (2×) histologic image overviews tissue condition at which the CIRPI system was used to collect radioluminescene and photoacoustic images (Fig. 1B). The histology slide illustrates a normal abdominal aortic wall thickness with no atherosclerotic plaques, highlighting the locations (i-iv) at which the CIRPI images were taken. A $360^{\circ}$ view of the radioluminescence image and all 4 quadrants showed no radioluminescent signal which renders an absence of macrophages (Supplemental Fig. 4A; Fig. 1C). Confirmatory clinical OCT images further validated these findings by showing normal abdominal aortic wall thickness with no atherosclerotic plaques (Fig. 1D). The highlighted $(3 \times)$ section in the OCT image showed no attenuation signals, a representation of no lipids or TCFA (Fig. 1E). For the photoacoustic image, no photoacoustic signals were detected at all 7 tested wavelengths, representing an absence of calcification, cholesterol cleft in the forms of cholesterol ester, phospholipids, cholesterol and triglyceride, and a presence of intact elastic fibers and collagen (Fig. 1F).

The area of the abdominal aorta of a diseased WHHL rabbit imaged with our CIRPI system is highlighted in the x-ray fluoroscopy image (Fig. 2A). Macrophages are found within the vulnerable plaques (TCFA, wall thickness $<65 \mu \mathrm{m}$ ) located on one side of the aortic wall of the in vivo WHHL rabbit based on both Trichrome/ H\&E-stained histologic slides (Figs. 2B, 2C-iv, and 2G). A high 


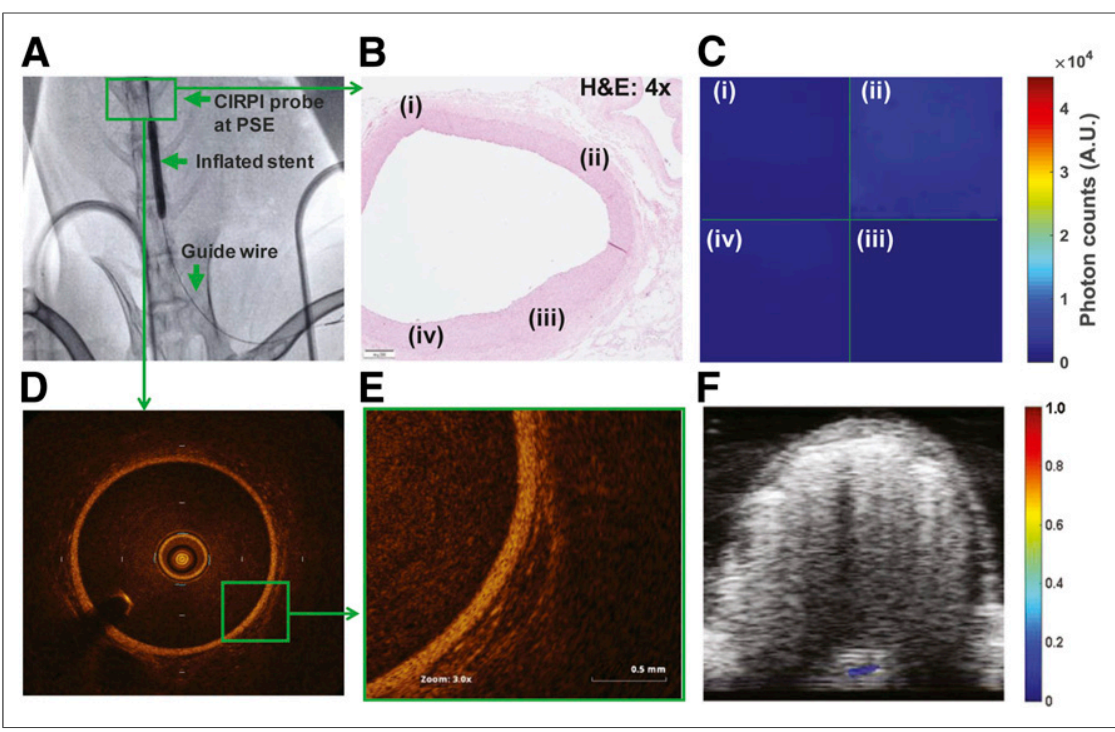

FIGURE 1. In vivo abdominal aorta images of the control rabbit. (A) x-ray-guided fluoroscopy image illustrates that a guide wire is followed by CIRPI probe located at the peripheral stent edge (proximal stent edge [PSE] is used as a landmark). (B) H\&E-stained (2x) histologic image overviews tissue condition at which CIRPI system is used to collect radioluminescene and photoacoustic images. Further, same location was imaged with a clinical OCT system for secondary verification. Histology slide illustrates normal abdominal aortic wall thickness with no atherosclerotic plaques. (C) Radioluminescence images are collected at every $1.43^{\circ}$ with scanning probe. Each quadrant (i), (ii), (iii), and (iv) is the representation of concatenated images between starting point of scan up to $90^{\circ}$. Each quadrant of radioluminescence image represents precise location of histologic slide that is highlighted with same number. None of the quadrant of radioluminescence image showed radioluminescent signal that renders an absence of macrophages. (D) Confirmatory OCT images further validate these findings by showing normal abdominal aortic wall thickness with no atherosclerotic plaques. (E) Highlighted section in OCT image shows no attenuation signals, a representation of no lipids or TCFA. (F) In photoacoustic image, no photoacoustic signals are detected at all 7 tested wavelengths, representing an absence of calcification, cholesterol cleft in form of cholesterol ester, phospholipids, cholesterol and triglyceride, and a presence of intact elastic fibers and collagen.

H\&E-stained histologic images (Figs. 3A and $3 \mathrm{~B})$. However, the clinical OCT system was unable to detect calcification at that location. The PAT image in Figure $3 \mathrm{C}$ was reconstructed from 5 individual PAT images representing 4 different lipid compositions and calcification and then superimposed on the ultrasound image.

Overall, our CIRPI system detected sparse $(n=2)$, moderate $(n=2)$, and severe $(n=1)$ macrophages in in vivo WHHL rabbits. Four of 5 WHHL rabbits had moderate-to-severe calcification and severe cholesterol clefts in the forms of cholesterol ester, phospholipids, cholesterol, and triglyceride. We found a 250 \pm 30.33 A.U. (arbitrary units; $P=0.001$ ) photoacoustic signal from non-TCFA at the 540- and 560-nm wavelengths, indicating calcification was present. However, no photoacoustic signal was detected in the TCFA for the 540-nm wavelength. We observed $240 \pm 20.56$ A.U. $(P=0.003)$, $110 \pm 8.67$ A.U. $(P=0.004), 210 \pm$ 15.85 A.U. $(P=0.002)$, and $90 \pm 5.75$ A.U. $(P=0.003)$ photoacoustic signals from lipids in the forms of cholesterol ester $(920 \mathrm{~nm})$, phospholipids $(1040 \mathrm{~nm})$, cholesterol $(1210 \mathrm{~nm})$, and triglyceride $(1235 \mathrm{~nm})$, respectively, in the area of TCFA. No photoacoustic signal was detected in the areas with non-TCFA. One of the WHHL rabbits showed early-stage atherosclerosis with no calcification or cholesterol clefts. This rabbit also showed

amount of radioluminescent signal is collected with our CIRPI probe at the location with macrophages within TCFA (Fig. 2D-iii; Supplemental Fig. 4B). Trichrome (2x) and H\&E (4X)-stained histologic images also highlighted stable (wall thickness $>65 \mu \mathrm{m}$ ) plaques (Figs. 2B and 2C-i, ii, iv). The clinical OCT system also confirms our findings with the CIRPI system and histochemical analysis (Figs. 2E and 2F). Statistical analysis showed $203 \times$ times higher radioluminescent signal (TCFA vs. non-TCFA: $6.36 \times 10^{4} \pm$ $5.3 \times 10^{3}$ vs. $3.14 \times 10^{1} \pm 1.91 \times 10^{1}$ photon counts, $P=0.003$ ) from the area with macrophages and lipids within the TCFA compared with stable plaques (Fig. $2 \mathrm{H}$ ).

We detected cholesterol cleft within TCFA with our CIRPI system in the forms of cholesterol ester, phospholipids, cholesterol, and triglyceride on collected photoacoustic signals at 920-, 1040-, 1210-, and 1235-nm wavelengths, respectively (Fig. 3C). $\mathrm{H} \& \mathrm{E}(4 \times)$-stained histologic images also confirmed these tissue compositions (Figs. 3A and 3B). Further, OCT image verified these results by showing an area of low attenuation, a representation of lipid/cholesterol within the TCFA (Fig. 3D). We observed photoacoustic signals from broken elastic fibers and damaged collagens at the 1180-nm wavelength with our CIRPI system (Fig. 3F). These findings were confirmed with EVG $(2 \times)$ (Fig. 3E)-stained histochemical image. Calcification was detected within stable plaques when our CIRPI system laser was tuned at the 540- and 560-nm wavelength (Fig. 3C). This finding was further confirmed with the intact elastic fibers and a small amount of collagen deposits. The other 4 WHHL rabbits exhibited broken elastic fibers and collagen deposits. Statistically, we found a $80 \pm 4.71$ A.U. $(P=0.0001)$ photoacoustic signal at the $1180-\mathrm{nm}$ wavelength that elucidated broken elastic fibers and collagens. The clinical OCT system showed early-stage atherosclerosis in 2 of $5 \mathrm{WHHL}$ rabbits with intimal hypertrophy. In addition, 4 of 5 WHHL rabbits exhibited lipid-filled TCFA with wall thickness between 40 and $60 \mu \mathrm{m}$, which were located within 7.9$30.9 \mathrm{~mm}$ from the proximal stent edge. One rabbit showed multiple TCFAs along with several stable plaques. Histology analysis corroborated these findings by showing occlusion between $10 \%$ and $60 \%$. Control rabbits showed normal abdominal aortic wall thickness with no plaques using our CIRPI system and verified with the clinical OCT system and histologic analysis. Results from each rabbit are shown in Supplemental Table 1.

\section{DISCUSSION}

We have demonstrated that our multimodality catheter-based CIRPI system can detect ${ }^{18} \mathrm{~F}$-FDG uptake by the macrophages within the TCFA of in vivo rabbit abdominal aorta with high resolution and sensitivity and is capable of simultaneously characterizing plaque morphologic compositions. These findings were verified with clinical OCT and ultrasound imaging systems, as well as immunohistochemical analyses of the molecular composition and pathologic status. The data based on radioluminescence 


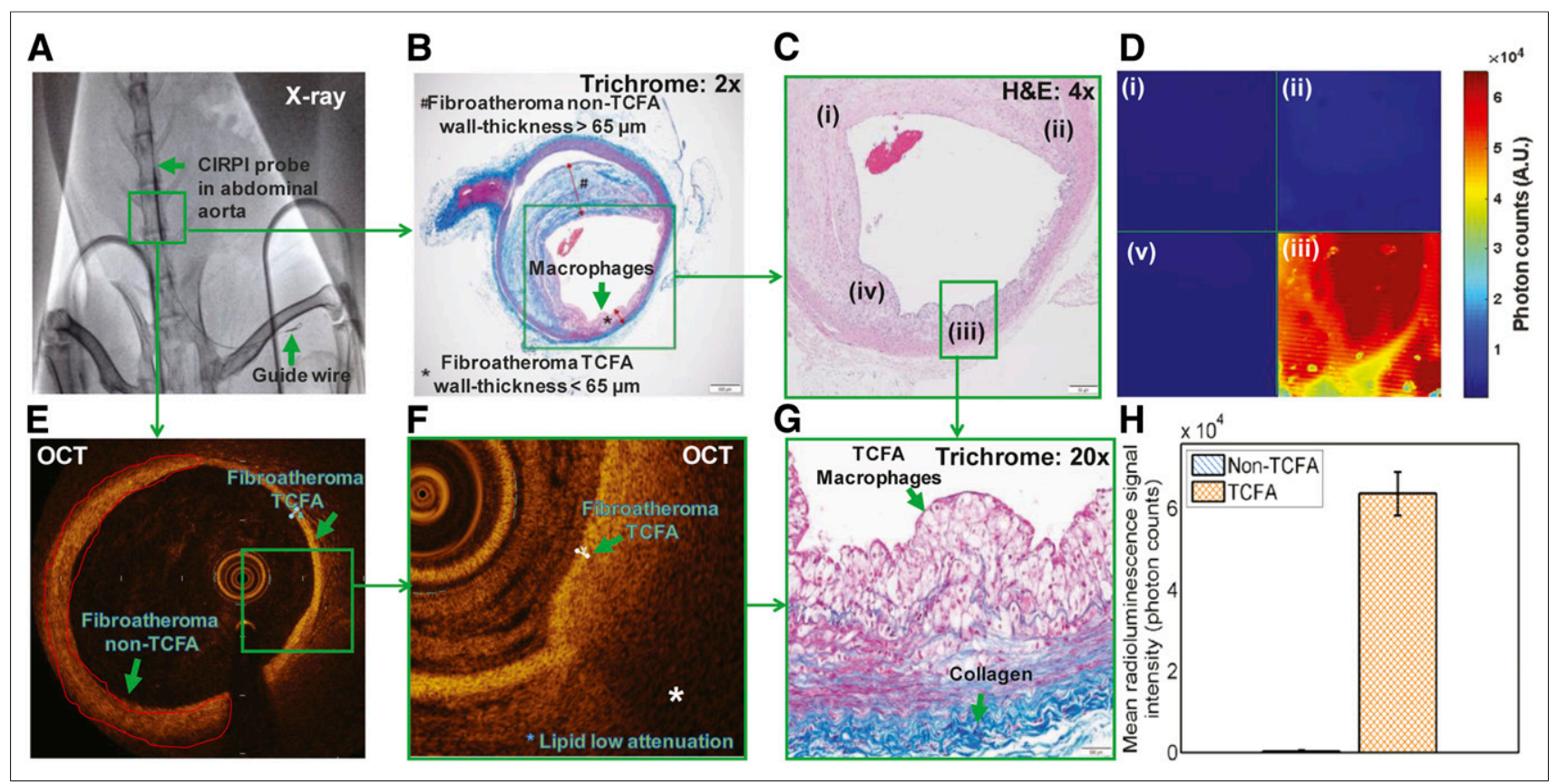

FIGURE 2. In vivo images of abdominal aorta with atherosclerotic plaques in WHHL rabbit. (A) x-ray-guided fluoroscopy image illustrates CIRP probe followed by guide wire marked with stent (highlighted within a green box). Histologic images are presented to give overview of plaque orientation within $\mathrm{CRI}$ imaging area using our CIRPI system and secondary verification with a clinical OCT system. (B) Trichrome-stained (2x) abdominal aorta highlights stable plaque, fibroatheroma non-TCFA with wall thickness $>65 \mu \mathrm{m}$. On the opposite wall there is clear evidence of fibroatheroma TCFA with a wall thickness $<65 \mu \mathrm{m}$ filled with macrophages. (C) H\&E-stained (4x) abdominal aorta shows 4 highlighted locations, (i), (ii), (iii), and (iv) in histology image that are same location of each quadrant of CIRPI image; (i), (ii), and (iii) represent non-TCFA, and (iv) TCFA with large macrophage accumulation. (D) Radioluminescence images are collected at every $1.43^{\circ}$ with scanning probe. Each quadrant is representation of concatenated images between starting point of scan up to $90^{\circ}$. Each quadrant of radioluminescence image represents precise location of histologic slide that is highlighted with same number. Quadrant iv of radioluminescence image exhibits high radioluminescent signal that is indicative of large macrophage accumulation within TCFA. (E) Confirmatory OCT images validated presence of non-TCFA and TCFA at arterial wall of abdominal aorta. (F) Highlighted section in OCT image shows low attenuation signal, a representation of lipids within TCFA. (G) Trichrome-stained TCFA area illustrates large number of macrophages that represents same area with lipids in OCT image. (H) Statistical analysis showed 203 $\times$ higher radioluminescent signal (TCFA vs. non-TCFA: $6.36 \times 104 \pm 5.3 \times 103$ vs. $3.14 \times 101 \pm 1.91 \times 101$ photon counts, $P=0.003$ ) from area with macrophages and lipids within TCFA compared with non-TCFA.

and photoacoustic signals illustrated low variability based on the statistical analysis. The radioluminescence signal variability among all disease rabbits was within $8.33 \%$. On the basis of the photoacoustic signals, the disease tissue compositions showed variabilities between $5.89 \%$ and $12.13 \%$ (calcification: $12.13 \%$, cholesterol ester: $8.56 \%$, phospholipids: $7.88 \%$, cholesterol: $7.54 \%$, triglyceride: $6.38 \%$, and elastin/collagen: $5.89 \%$ ). The lowest variability was observed for elastin/collagen whereas calfication varied the most. We were not able to discuss these results in ratio as we found no photoacoustic signal for calcification (540 and $560 \mathrm{~nm}$ ) from TCFA and cholesterol ester, phospholipids, cholesterol, triglyceride, and broken elastic fibers/collagen from non-TCFA.

Our integrated CIRPI system offers an unprecedented opportunity for intravascular detection and characterization of the biology and morphology of atherosclerotic plaques that provides a multiparametric analysis of pathognomonic constituents of vulnerable plaques. This approach will both broaden our understanding about human CAD and enable prognostic data for this deadly disease. Such measurements have important implications for guiding treatment that may ultimately lead to reducing the risk of heart attack and the debilitating aftermath.

On the basis of the results analysis, we found that if the aortic occlusion is less than $10 \%$, the plaques are mostly early stage with moderate macrophages. Also, these early stage atherosclerotic plaques have no calcification or cholesterol clefts, and its elastic fibers are still intact with a small amount of collagen deposition. When the aortic occlusion was between $25 \%$ and $60 \%$, we identified severe macrophages, calcifications, and cholesterol clefts in the form of cholesterol ester, phospholipids, cholesterol, and triglyceride located over a $500-\mu \mathrm{m}$ to $1-\mathrm{mm}$ area. In some rabbits, multiple TCFAs and stable plaques were identified in close vicinity. Most of these severe atherosclerotic abdominal aortas of the WHHL rabbits revealed broken elastic fiber and collagen deposition. The endogenous chromophores such as calcification, lipids cause the enhanced signal-to-background ratios.

Our CIRPI system uses an intravascular molecular imaging approach with optical, photoacoustic imaging in conjunction with ${ }^{18} \mathrm{~F}-\mathrm{FDG}$. The system collected radioluminescent and photoacoustic signals to detect the exact location of TCFA and demonstrated its usefulness for disease characterization through biologic imaging of atherosclerotic plaques. On the basis of this feasibility study, we demonstrated that our multimodality CIRPI prototype system was sufficiently sensitive toward the detection of ${ }^{18} \mathrm{~F}-\mathrm{FDG}$ in in vivo TCFA with good resolution. Thus, our novel system in conjunction with radionuclide molecular probes may lead to intravascular imaging platforms for coronary arterial molecular imaging in the clinic.

Coronary imaging remains challenging with PET or other currently available imaging modalities due to small size, constant 


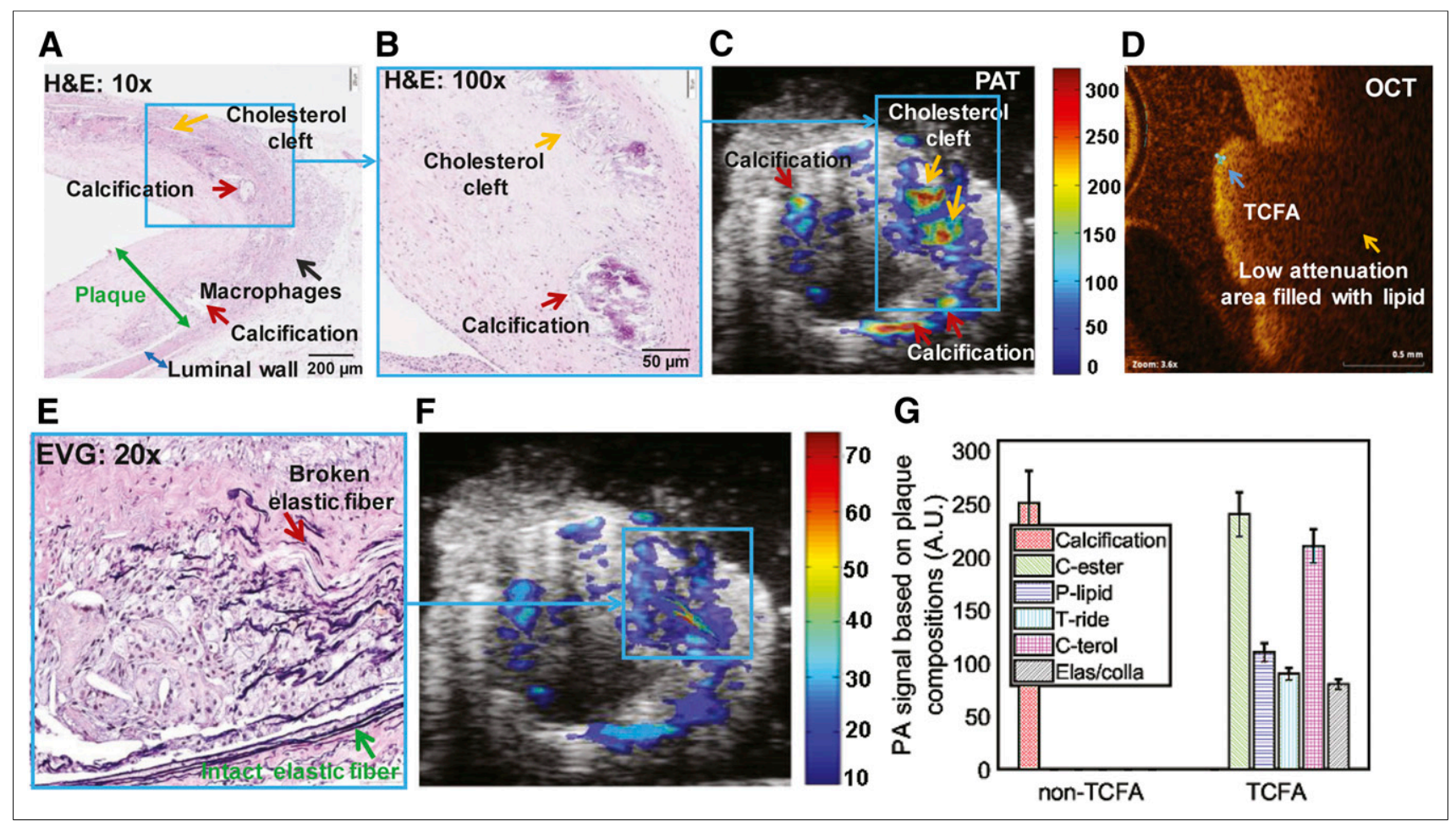

FIGURE 3. Images of in vivo atherosclerotic plaques in rabbit abdominal aorta. (A) Histologic images are presented to give overview of plaque orientation within PAT imaging area using our CIRPI system and secondary verification with clinical OCT system. H\&E-stained (10x) abdominal aorta highlighted plaques containing trace amount of calcification and prominent cholesterol cleft/lipids in close vicinity to macrophages, fibroatheroma TCFA with wall thickness $<65 \mu \mathrm{m}$. (B) Enlarged image of A shows severe cholesterol cleft and trace amount of calcification. (C) In PAT image, high photoacoustic signals were detected at area with cholesterol, calcification. (D) Confirmatory OCT images validated presence of presence of lipids at arterial wall of abdominal aorta. Highlighted section in OCT image showed low attenuation signal, a representation of lipids within TCFA. (E) EVG (20x)-stained area illustrates both broken (red arrow) and intact collagen (green arrow). (F) High photoacoustic signal was detected at area with broken collagen in PAT image collected with CIRPI system. (G) Statistical analysis showed high photoacoustic signal from (i) non-TCFA calcification (250 $\pm 30.33, P=0.001$, no other signal was observed); (ii) TCFA: cholesterol ester ( $240 \pm 20.56, P=0.003$ ), phospholipids (110 $\pm 8.67, P=0.004)$, cholesterol $(210 \pm 15.85, P=0.002)$, and triglyceride $(90 \pm 5.75, P=0.003)$, which represent the presence of severe lipids; elastin/collagen ( $80 \pm$ $4.71, P=0.0001$ ) that elucidates broken/damaged elastic fibers and collagen.

motion, and in the case of ${ }^{18} \mathrm{~F}-\mathrm{FDG}$, the obscuring of uptake by adjacent myocardium (27). Although ${ }^{18} \mathrm{~F}$-FDG uptake is a marker for viable myocardium, this myocardial uptake makes it harder to image the adjacent small-sized coronaries (28), though there are some promising initial results (29). This makes an intravascular imaging approach advantageous, minimizing the distance and maximizing the sensitivity to coronary plaque signal. Although ${ }^{18}$ F-FDG PET has been well studied, there are other PET agents being investigated, such as ${ }^{18} \mathrm{~F}$-labeled mannose (30), which could also be studied in the future by our CIRPI system.

As any scintillating screen with a thickness as small as $1 \mathrm{~mm}$ provides an intrinsic efficiency close to $10 \%$ due to annihilation photons, we chose to use a scintillating screen made of $\mathrm{CaF}_{2}: \mathrm{Eu}$ with a thickness of $0.150 \mathrm{~mm}$. Because of this extremely thin screen, the CIRPI system eliminates a significant amount of annihilation photons, which would have come from distant tissues such as the bladder caused by high $\gamma$-radiation from urine or $\gamma$-contamination that is spill in from the myocardium and hinders the imaging of the local ${ }^{18} \mathrm{~F}-\mathrm{FDG}$ uptake in the plaque. Also, this thin scintillating imaging window allows a small fraction of energy deposition from the $\gamma$-particles due to Compton interaction. Therefore, it was unlikely that the $\gamma$-particles would have interacted with the scintillator to create background noise. Thus, subtraction of the $\gamma$-radiation signal was not considered. We used a typical radiation dose, despite the nature of this feasibility study of the CIRPI system for in vivo imaging.

In this study, we have developed the central hypothesis on detecting and characterizing lipid-rich plaques using our CIRPI system through sufficiently well-differentiated absorption spectra of lipids from those of the constituents of normal arterial tissue in the 740- to $1400-\mathrm{nm}$ wavelength range. Although, we observed cholesterol clefts in the forms of cholesterol ester, phospholipid, cholesterol, and triglyceride in in vivo rabbit abdominal aorta with our CIRPI system, we could not confirm the presence of individual components of lipids with the histochemical analysis and clinical OCT system (histology and OCT cannot distinguish specific lipid components). However, we were able to correlate large lipid pools at the TCFA with both histology and the clinical OCT system. Thus, downscaling photoacoustic imaging of the CIRPI system provides a tool for understanding metabolic pathways. Because hypermetabolism is a quintessential hallmark of TCFA atherosclerotic plaques, metabolic photoacoustic imaging may enable clinical CAD screening at the earliest stage without using exogenous contrast agents.

Several intravascular hybrid imaging systems including IVISXray, OCT-Xray, IVUS-CTCA, OCT-CTCA, NIRS-IVUS, IVUS-OCT, OCT-NIRF, IVUS-NIRF, OCT-NIRS, IVUS-IVPA, and IVUS-FLIM 
were developed over the years to detect and evaluate atherosclerotic plaques. These systems were evaluated on the basis of their ability to get information on lumen dimension, plaque burden, positive remodeling, lipid content, and cap thickness. These hybrid imaging systems mentioned here have the ability to perform 3 of these areas efficiently, unlike our CIRPI system, which can perform all 5 . In these hybrid systems, NIRS-IVUS (3$16 \mathrm{~mm})$, IVUS-OCT (1 mm), OCT-NIRF (1-3 mm), OCT-NIRS $(1.5 \mathrm{~mm})$, and IVUS-IVPA $(4-5 \mathrm{~mm})$ are considered the best with respect to millimeter range resolution. However, none of the imaging systems has the resolution in $\mu \mathrm{m}$ as our CIRPI system $(1.2 \mu \mathrm{m})$ does. Acquisition time varies among all hybrid systems specially IVIS-OCT (20-45 s/image), OCT-NIRF (44 ms/image), and OCT-NIRS (2.5-4 min/image). These 3 hybrid systems have the best acquisition time compared with other hybrid imaging systems mentioned here. Our CIRPI system has the acquisition time of $2 \mathrm{~min} / \mathrm{image}$. Thus, the acquisition time of our CIRPI system is comparable with the top 3 intravascular hybrid imaging systems that are discussed in this article.

It is important to evaluate our CIRPI system sensitivity with respect to other intravascular $\beta$-detecting scintillator-based (BC-408 plastic) imaging systems such as intravascular radiation detector (IVRD) (31). However, it is almost impossible to compare the CIRPI system with other imaging modalities as this is the first time, to our knowledge, that $\mathrm{CaF}_{2}$ :Eu phosphor was ever used to make an imaging window for a catheter-based system. In addition, IVRD was used to collect $\beta$-particles from 115,230 , and $1,335 \mathrm{kBq}$ of ${ }^{18} \mathrm{~F}-\mathrm{FDG}$ point-fixed sources in the femoral artery of a dog instead of intravenous injection. The IVRD probe consists of only 100 optical fibers that can only create spectral images, unlike our CIRPI system that has 18,000 optical image fibers to create a $360^{\circ}$ image of the artery.

We found that our design of the CIRPI system is suitable for early detection/characterization of vulnerable plaque with high sensitivity/ accuracy and to track the plaque biology/morphology by multiparametric analysis of pathognomonic compositions of TCFA. This approach can both broaden our understanding about human CAD and enable prognostic data for this deadly disease. Therefore, such measurements will have important implications for guiding treatment that may ultimately lead to reduce the risk of MI and the aftermath. Thus, this CIRPI system may propose a paradigm shift in the diagnosis and risk stratification of CAD. The synergy that would be created by this imaging system could unite molecular and pathologic information associated with TCFA. Therefore, our CIRPI system with advanced catheter design can reveal molecular aspects of plaque pathobiology that may constitute a valuable and indispensable ally in this challenging quest.

The CIRPI system catheter probe needs to be within 1-3 mm of a vulnerable plaque to capture signal from the $\beta$-particle emitting from ${ }^{18} \mathrm{~F}$-FDG decay despite the field of view of $6.5 \mathrm{~mm}$. This close distance between the plaques and probe may increase the risk of rupturing TCFA. Another limitation to the system is the probe's rigid scanning head as it limited the bending radius of the probe. In the future, we plan to reduce the length of the rigid head with decrease the radius.

Despite these limitations with the current CIRPI system, we demonstrate future potential for clinical detection, diagnosis, and treatment of human coronary atherosclerosis and offer the possibilities for expanding our understanding of CAD. The CIRPI system was designed with essential features that will enable intravascular imaging for clinical characterization of vulnerable coronary plaques and is destined to have an impact on how we manage this disease.

\section{CONCLUSION}

Although significant advancements have been made in medical imaging, CAD remains the number one cause of death in the United States and worldwide. Early detection of atherosclerosis and prevention of myocardial infarction and sudden cardiac death have remained critical unmet medical needs, which we address using this multimodality imaging approach. In the last $15 \mathrm{y}$, molecular imaging strategies have become an integral part of basic science and recently in the clinical diagnosis of numerous disease. However, there remains a substantial translational gap for molecular imaging of coronary atherosclerosis in patients. Our multimodality molecular tissue imaging approach comprises an integrated fiber-optic CIRPI system, harnessing light, radioluminescence, and sound to detect and characterize vulnerable atherosclerotic plaques. We could outline the plaque location with characteristic information pertaining to specific tissue compositions. Our catheter-based system provides the high sensitivity and resolution needed for coronary plaque imaging. Radioluminescence and photoacoustic imaging are ripe for clinical translation, particularly as there are multiple Food and Drug Administration-approved radionuclides (e.g., ${ }^{18}$ F-FDG). We demonstrated the potential for clinical detection and evaluation of in vivo vulnerable and nonvulnerable atherosclerotic plaques via CIRPI, plus the potential for expanding our understanding of CAD to ultimately improve how we manage this disease.

\section{DISCLOSURE}

We gratefully acknowledge the following funding agencies for supporting this research work: this study was fully supported by the NIH K99/R00 award (1 K99 HL127180-01) and partly supported by NIH Shared Instrumentation Grant (S10OD010344-01A1). Michael V. McConnell is currently a clinical professor at the Stanford University School of Medicine and an employee of Verily Life Sciences; however, this does not alter our adherence to the Nature Biomedical Engineering policies on sharing data and materials. No other potential conflict of interest relevant to this article was reported.

\section{ACKNOWLEDGMENT}

We gratefully acknowledge Jennifer Lyons for her help with the in vivo rabbit surgery.

\section{KEY POINTS}

QUESTION: Can the CIRPI system overcome the current clinical system's limitation in detecting thin-cap fibroatheroma (TCFA)?

PERTINENT FINDINGS: Early stage TCFA showed macrophages. Advanced-stage TCFA showed broken elastic fibers/collagen, lipids with one-to-one correlation with histology. Results were statistically significant.

IMPLICATIONS FOR PATIENT CARE: These results demonstrated the potential for clinical detection and evaluation of vulnerable and nonvulnerable atherosclerotic plaques via CIRPI.

\section{REFERENCES}

1. Go AS, Mozaffarian D, Roger VL, et al. Executive summary: heart disease and stroke statistics-2014 update-a report from the American Heart Association. Circulation. 2014;129:399-410. 
2. Ross R. The pathogenesis of atherosclerosis: a perspective for the 1990s. Nature. 1993;362:801-809.

3. Ross R. Atherosclerosis: an inflammatory disease. N Engl J Med. 1999;340:115-126.

4. Garcia-Garcia HM, Costa MA, Serruys PW. Imaging of coronary atherosclerosis: intravascular ultrasound. Eur Heart J. 2010;31:2456-2469.

5. Chen IY, Wu JC. Cardiovascular molecular imaging: focus on clinical translation. Circulation. 2011;123:425-443.

6. Jang IK, Tearney GJ, MacNeill B, et al. In vivo characterization of coronary atherosclerotic plaque by use of optical coherence tomography. Circulation. 2005;111:1551-1555.

7. Yabushita H, Bouma BE, Houser SL, et al. Characterization of human atherosclerosis by optical coherence tomography. Circulation. 2002;106:1640-1645.

8. Calfon MA, Vinegoni C, Ntziachristos V, Jaffer FA. Intravascular near-infrared fluorescence molecular imaging of atherosclerosis: toward coronary arterial visualization of biologically high-risk plaques. J Biomed Opt. 2010;15:011107.

9. Kim JW, Jaffer FA. Emerging molecular targets for intravascular imaging of high-risk plaques. Curr Cardiovasc Imaging Rep. 2010;3:237-247.

10. Sanz J, Fayad ZA. Imaging of atherosclerotic cardiovascular disease. Nature. 2008;451:953-957.

11. Jaffer FA, Libby P, Weissleder R. Molecular imaging of cardiovascular disease. Circulation. 2007;116:1052-1061.

12. Wang B, Yantsen E, Larson T, et al. Plasmonic intravascular photoacoustic imaging for detection of macrophages in atherosclerotic plaques. Nano Lett. 2009;9:2212-2217.

13. Yeager D, Karpiouk A, Wang B, et al. Intravascular photoacoustic imaging of exogenously labeled atherosclerotic plaque through luminal blood. J Biomed Opt. 2012;17:106016.

14. Jansen K, van Soest G, van der Steen AF. Intravascular photoacoustic imaging: a new tool for vulnerable plaque identification. Ultrasound Med Biol. 2014;40:12.

15. Groen HC, Gijsen FJ, van der Lugt A, et al. Plaque rupture in the carotid artery is localized at the high shear stress region: a case report. Stroke. 2007;38:2379-2381.

16. Hyafil F, Cornily JC, Feig JE, et al. Noninvasive detection of macrophages using a nanoparticulate contrast agent for computed tomography. Nat Med. 2007;13: 636-641.

17. McLachlan SJ, Morris MR, Lucas MA, et al. Phase I clinical evaluation of a new iron oxide MR contrast agent. J Magn Reson Imaging. 1994;4:301-307.

18. Tang TY, Howarth SP, Miller SR, et al. The ATHEROMA (Atorvastatin Therapy: Effects on Reduction of Macrophage Activity) Study: evaluation using ultrasmall superparamagnetic iron oxide-enhanced magnetic resonance imaging in carotid disease. J Am Coll Cardiol. 2009;53:2039-2050.

19. Dimastromatteo J, Broisat A, Perret P, et al. In vivo molecular imaging of atherosclerotic lesions in ApoE-/- mice using VCAM-1-specific, ${ }^{99 \mathrm{~m}} \mathrm{Tc}$ labeled peptidic sequences. J Nucl Med. 2013;54:1442-1449.

20. Zhu Q, Piao D, Sadeghi MM, Sinusas AJ. Simultaneous optical coherence tomography imaging and beta particle detection. Opt Lett. 2003;28:1704-1706.

21. Moreno PR. Vulnerable plaque: definition, diagnosis, and treatment. Cardiol Clin. 2010;28:1-30.

22. Bourantas CV, Kourtis IC, Plissiti ME, et al. A method for $3 \mathrm{D}$ reconstruction of coronary arteries using biplane angiography and intravascular ultrasound images. Comput Med Imaging Graph. 2005;29:597-606.

23. García-García HM, Gonzalo N, Granada JF, Regar E, Serruys PW. Diagnosis and treatment of coronary vulnerable plaques. Expert Rev Cardiovasc Ther. 2008;6: 209-222.

24. Zaman RT, Yousefi S, Long SR, et al. A dual-modality hybrid imaging system harnesses radioluminescence and sound to reveal molecular pathology of atherosclerotic plaques. Sci Rep. 2018;8:8992.

25. Zaman RT, Kosuge H, Carpenter C, Sun C, McConnell MV, Xing L. Scintillating balloon-enabled fiber-optic system for radionuclide imaging of atherosclerotic plaques. J Nucl Med. 2015;56:771-777.

26. Zaman RT, Yousefi S, Long S, et al. Harnessing radioluminescence and sound to reveal molecular pathology of atherosclerotic plaques. J Nucl Med. 2017; 58(suppl 1):31.

27. Gaeta C, Fernandez Y, Pavia J, et al. Reduced myocardial ${ }^{18}$ F-FDG uptake after calcium channel blocker administration: initial observation for a potential new method to improve plaque detection. Eur J Nucl Med Mol Imaging. 2011;38: 2018-2024.

28. Inglese E, Leva L, Matheoud R, et al. Spatial and temporal heterogeneity of regional myocardial uptake in patients without heart disease under fasting conditions on repeated whole-body ${ }^{18}$ F-FDG PET/CT. J Nucl Med. 2007;48:1662-1669.

29. Rogers IS, Nasir K, Figueroa AL, et al. Feasibility of FDG imaging of the coronary arteries: comparison between acute coronary syndrome and stable angina. JACC Cardiovasc Imaging. 2010;3:388-397.

30. Tahara N, Mukherjee J, de Haas HJ, et al. 2-deoxy-2-[18F]fluoro-D-mannose positron emission tomography imaging in atherosclerosis. Nat Med. 2014;20:215-219.

31. Hosokawa R, Kambara N, Ohba M, et al. A catheter-based intravascular radiation detector of vulnerable plaques. J Nucl Med. 2006;47:863-867. 